\title{
Static characteristic analysis of equivalent spring element of the triangle constant element
}

\author{
Hong-Ye Su ${ }^{1, a}$, YangZhao ${ }^{2, b}$, GeTian $^{3, \mathrm{c}}$, Feng Shen ${ }^{4, \mathrm{~d}}$,Sheng-Jun Zhang ${ }^{5, \mathrm{e}}$, \\ Xiang-Rong $\mathrm{Fu}^{6, f^{*}}$ \\ ${ }^{1}$ Department of civil Engineering, China Agricultural University, Beijing, 100083 China \\ ${ }^{2}$ China Nuclear Power Engineering Co., Ltd., Beijing, 100840 China \\ ${ }^{3}$ Department of civil Engineering, China Agricultural University, Beijing, 100083 China \\ ${ }^{4}$ State power investment central research institute , Beijing, 102209 China \\ ${ }^{5}$ State power investment central research institute., Beijing, 102209 China \\ ${ }^{6}$ Department of civil Engineering, China Agricultural University, Beijing, 100083 China \\ aSuhy@cau.edu.cn, bzhaoyang@cnpe.cc, ctracytiange@163.com, ${ }^{\mathrm{d}}$ shenfeng@snptc.com.cn, \\ ezhangshengjun@snptc.com.cn,fuxr@cau.edu.cn
}

Keywords:finite element,equivalent spring element,continuous medium.

Abstract. In this paper, the equivalent mechanical spring model of the three-dimensional continuumis studied. And the sectional area of the equivalent spring element is obtained based on the displacement equivalent principle. The equivalent model is statically indeterminate trusses system of spring. The 3D spring calculation model can be used in the static characteristic analysis. By using equivalent spring element model as the basic unit of analysis of a triangle continuum static analysis problems, and with the use of triangle constant element as basic element income results were compared. The results show that the simplified form in good agreement with the exact solution, the establishment of the equivalent, and compared with the commonly used continuous medium finite element model, computational efficiency and accuracy of equivalent spring element model is higher. It shows that the equivalent spring model have theequivalent stiffness in the static analysis.

\section{Introduction}

At present, the vast majority of structure design and analysis of software engineering is based on finite element theory, which makes the high precision and high stability of the finite element calculation model research become a hot issue. In this paper, a class of equivalent spring element theory model is proposed based on the displacement equivalence principle. Due to the existence of frame structure calculation theory more perfect, so in the calculation software and the universal application in engineering analysis before continuous medium shell or entity structure transformation for the equivalent system of pole is calculated as a hot research topic.

Based on the above ideas, this paper makes a theoretical study on the equivalent spring model of triangular continuous medium, and an equivalent spring element model is obtained by the principle of displacement equivalent. At the same time, the problem of the deformation displacement of the triangle plate is verified by numerical examples.

\section{The calculation method of the equivalent truss model.}

The model of continuous media element is showed in Fig.1, where $a, b$, and c represent side lengths of the triangular. The mechanical analysis of statically indeterminate truss structure with the same size is showed in Fig.2. The stiffness of spring can be derived from the axial stiffness of truss ${ }^{[2]}$. 


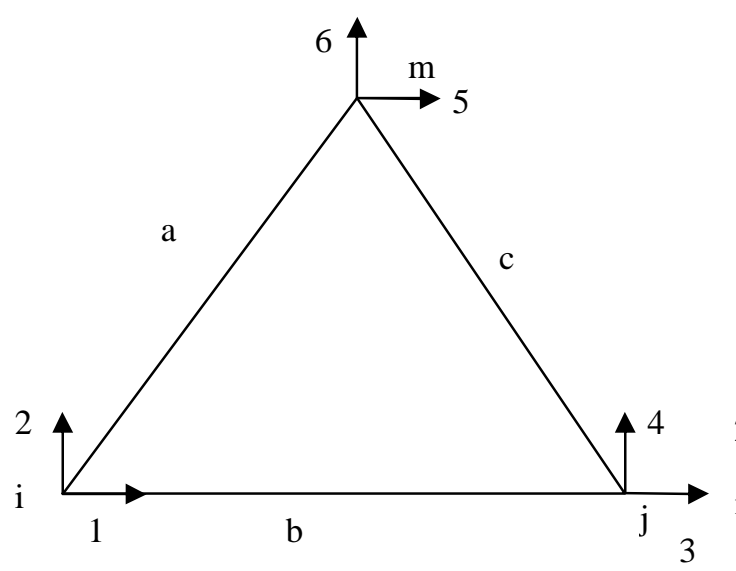

Fig.1 The CST element

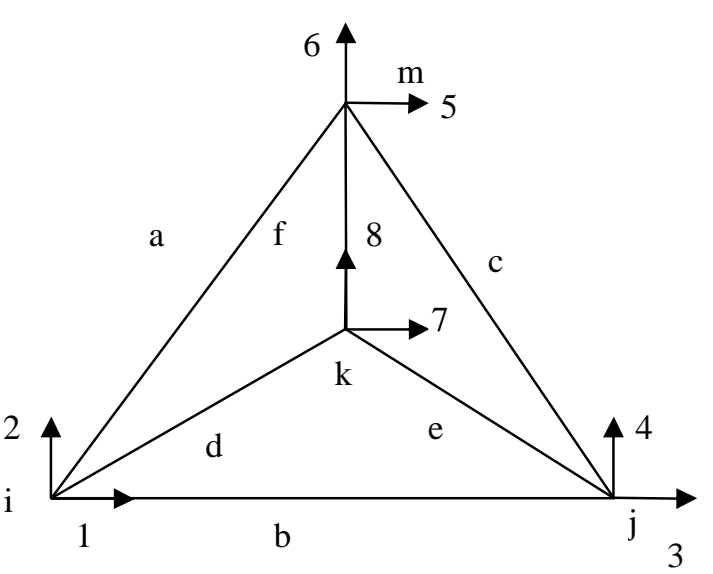

Fig.2 The final simplified model

The three nodes are $\mathrm{i}, \mathrm{j}, \mathrm{m}$, and the triangle center is $\mathrm{K}$. The rod length is $\mathrm{a}, \mathrm{b}, \mathrm{c}, \mathrm{d}, \mathrm{e}, \mathrm{f}$. The bar section area is $A_{1}, A_{2}, A_{3}, A_{4}, A_{5}, A_{6}$.

The symmetry of the isosceles triangle shows that the stiffness matrix can be represented by three relatively independent variables. The three independent variable are $\alpha_{3}, \alpha_{4}, \alpha_{5}$. The stiffness matrix is expressed as follows.

$$
\left[\begin{array}{cccccc}
-\alpha_{3}-\alpha_{5} & \alpha_{4}-\frac{2 \mathrm{~h}}{\mathrm{~b}} \alpha_{5} & \alpha_{3} & \alpha_{4} & \alpha_{5} & -2 \alpha_{4}+\frac{2 h}{b} \alpha_{5} \\
-\left(\frac{2(f-h)}{b} \alpha_{4}+\frac{4 h^{2}}{b^{2}} \alpha_{5}\right) & -\alpha_{4} & -\frac{2(f-h)}{b} \alpha_{4} & \frac{2 \sqrt{a^{2}-\frac{b^{2}}{4}}}{b} \alpha_{5} & \frac{4(f-h)}{b} \alpha_{4}+\frac{4 h^{2}}{b^{2}} \alpha_{5} \\
-\alpha_{3}-\alpha_{5} & -\left(\alpha_{4}-\frac{2 h}{b} \alpha_{5}\right) & \alpha_{5} & -\left(-2 \alpha_{4}+\frac{2 h}{b} \alpha_{5}\right) \\
& -\left(\frac{2(f-h)}{b} \alpha_{4}+\frac{4 h^{2}}{b^{2}} \alpha_{5}\right) & -\frac{2 \sqrt{a^{2}-\frac{b^{2}}{4}}}{b} \alpha_{5} & \frac{4(f-h)}{b} \alpha_{4}+\frac{4 h^{2}}{b^{2}} \alpha_{5} \\
& & -2 \alpha_{5} & 0 \\
& & & -2\left(\frac{4(f-h)}{b} \alpha_{4}+\frac{4 h^{2}}{b^{2}} \alpha_{5}\right)
\end{array}\right]
$$

Three independent variables of the constant stress element are :

$$
\begin{aligned}
& \alpha_{3}=\mathrm{K}_{13}=\frac{\mathrm{Et}\left[\mathrm{b}^{2}(\mu-3)+8 a^{2}\right]}{32 \mathrm{~A}\left(\mu^{2}-1\right)} \\
& \alpha_{4}=\mathrm{K}_{14}=-\frac{\operatorname{Etb}(3 \mu-1) \sqrt{4 \mathrm{a}^{2}-\mathrm{b}^{2}}}{32 \mathrm{~A}\left(\mu^{2}-1\right)} \\
& \alpha_{5}=\mathrm{K}_{15}=-\frac{\mathrm{Etb}^{2}}{16 \mathrm{~A}(\mu+1)}
\end{aligned}
$$

Three independent variables of the spring element are :

$$
\begin{aligned}
& \alpha_{3}=\mathrm{K}_{\mathrm{s} 13}=-\frac{\mathrm{Eb}^{2} \mathrm{~A}_{4} \mathrm{~A}_{6}}{8\left(\mathrm{~A}_{6} \mathrm{~d}^{3}+2 \mathrm{~A}_{4} \mathrm{f}^{3}-4 \mathrm{~A}_{4} \mathrm{hf}^{2}+2 \mathrm{~A}_{4} \mathrm{fh}^{2}\right)}-\frac{E A_{2}}{b} \\
& \alpha_{4}=\mathrm{K}_{\mathrm{s} 14}=-\frac{\mathrm{EbA}_{4} \mathrm{~A}_{6}(f-h)}{4\left(\mathrm{~A}_{6} \mathrm{~d}^{3}+2 \mathrm{~A}_{4} \mathrm{f}^{3}-4 \mathrm{~A}_{4} \mathrm{hf}^{2}+2 \mathrm{~A}_{4} \mathrm{fh}^{2}\right)} \\
& \alpha_{5}=\mathrm{K}_{\mathrm{s} 15}=-\frac{\mathrm{EA}_{1} \mathrm{~b}^{2}}{4 \mathrm{a}^{3}}
\end{aligned}
$$

Among them, $\mathrm{K}_{13}, \mathrm{~K}_{14}, \mathrm{~K}_{15}, \mathrm{~K}_{\mathrm{s} 13}, \mathrm{~K}_{\mathrm{s} 14}, \mathrm{~K}_{\mathrm{s} 15}$, are Stiffness。 
The introduction of simplified formulas $\mathrm{f}$ :

$$
f=h-\frac{\mathrm{b} \cdot \mathrm{K}_{24}}{2 \cdot \mathrm{K}_{14}} \quad f=\frac{2 \mu h^{2}-\frac{b^{2}}{2}}{h(3 \mu-1)}
$$

The reasonable range of $\mathrm{f}$ is $(0, \mathrm{~h})$.

$$
\begin{aligned}
& 0<\frac{2 \mu h^{2}-\frac{b^{2}}{2}}{h(3 \mu-1)}<h \\
& \left\{\begin{array}{c}
\mu>\frac{1}{3}, 2 h^{2}(1-\mu)<b^{2}<4 \mu h^{2} \\
0<\mu<\frac{1}{3}, 2 h^{2}(1-\mu)>b^{2}>4 \mu h^{2}
\end{array}\right.
\end{aligned}
$$

When $\mu=\frac{1}{3}$,it is a special case.

the line chart as follows :

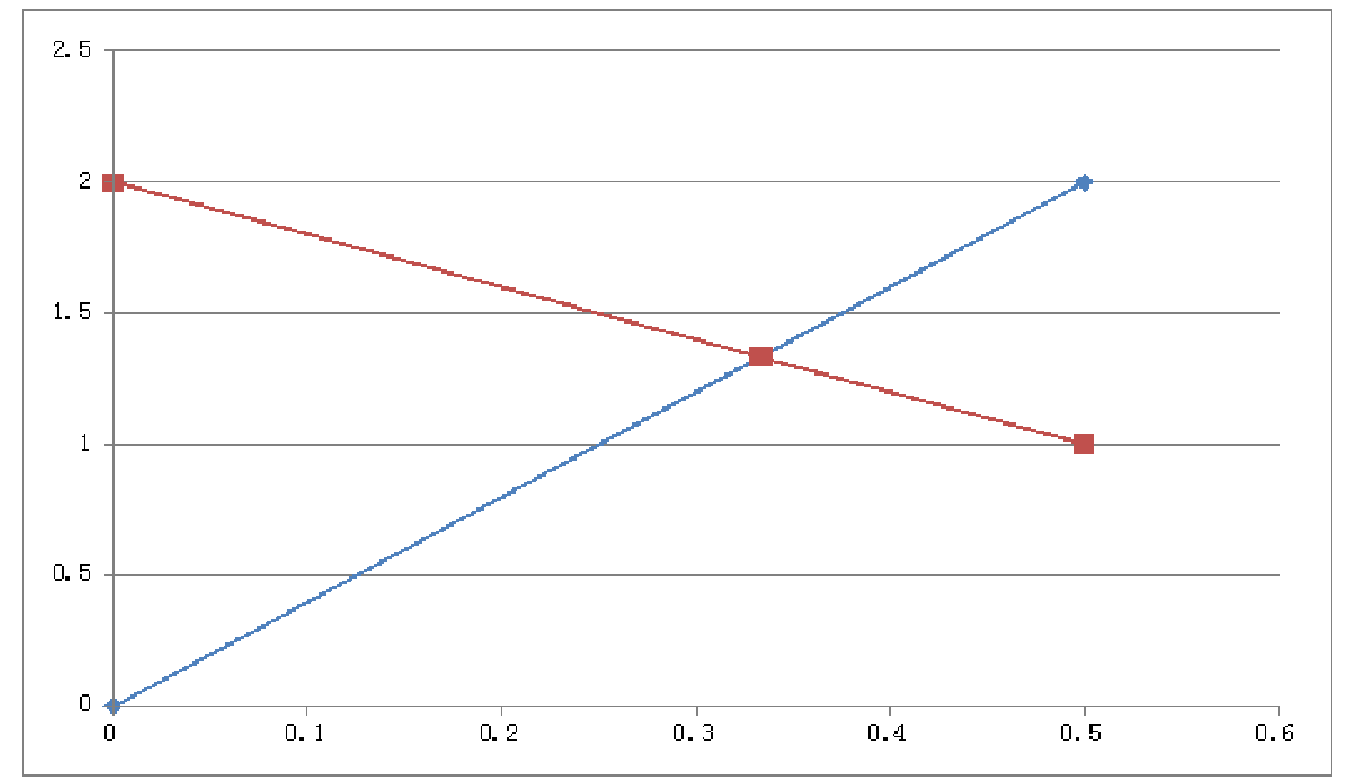

Note:The middle area of two lines is the reasonable range.

Considering $\alpha_{5}$, we can get $A_{1}=\frac{a^{3} t}{4 A(\mu+1)}$.

Considering $\alpha_{3}, \alpha_{4}$, we can get $\mathrm{A}_{2}=\frac{\mathrm{bt}\left[\mathrm{b}^{2}(\mu-3) f+2 \mathrm{~b}^{2} h(1+\mu)+8 \mathrm{a}^{2}(f-h)\right]}{32 \mathrm{~A}\left(1-\mu^{2}\right)(\mathrm{f}-\mathrm{h})}$.

Because of the characteristics of constant stress unit,equivalent spring element needs to meet the conditions $\varepsilon_{f}=\varepsilon_{d}$.

$$
\varepsilon_{f}=\frac{1}{E A_{6}}, \varepsilon_{d}=\frac{d}{2(h-f) E A_{4}}
$$

We can get $A_{6}=\frac{2(h-f)}{d} A_{4}$.

Considering $\alpha_{4}$, we can get $A_{6}=\frac{\left(2 f^{3}-4 f^{2} h+2 f h^{2}\right) t(3 \mu-1) h A_{4}}{4 A_{4} A\left(\mu^{2}-1\right)(f-h)-d^{3} t(3 \mu-1) h}$. 


$$
\text { So } \begin{aligned}
A_{4} & =\frac{\operatorname{th}(3 \mu-1)\left[d^{3}+d f(h-f)\right]}{4 A\left(\mu^{2}-1\right)(f-h)} \\
A_{6} & =\frac{\operatorname{th}(3 \mu-1)\left[d^{2}+f(h-f)\right]}{2 A\left(1-\mu^{2}\right)}
\end{aligned}
$$

\section{Conclusion}

In this paper, a model of continuous medium equivalent to spring element is obtained by means of stiffness equivalence principle. The simplification of the structure of the triangle continuous medium to the rod system is accomplished. A numerical example is carried out to verify the results. The results indicate that the equivalent was established.

\section{Acknowledgement}

the National Natural Science Foundation of China (Nos.11272340)

National Science and Technology Major Project of the Ministry of Science and Technology of China(No. 2015ZX06004004-003)

\section{References}

[1] Fu, X. R. and Long, Y. Q. Generalized conforming quadrilateral plane elements based on analytical trial functions (in Chinese). Engineering Mechanics, 19(4), 12-16 (2002)

[2] Zhang Chong, Jin Feng ,HouYanli, 3-D simple deformable distinct elementmethod,Chinese journal of geotechnical engineering , , 2007,29 (2) : 159-163.

[3] Long Yuqiu ,BaoShihua,KuangWenqi, Yuansi, structure mechanics[M],Higher education 2012

[4] Fu, X. R., Cen, S., Long, Y. Q., Jiang X. G., and Ju, J. S. The analytical trial function method (ATFM) for finite element analysis of plane crack/notch problems. Key Engineering Materials, 385-387, 617-620 (2008)

[5] Fu, X. R., Cen, S., Li, C. F., and Chen, X. M. Analytical trial function method for development of new 8-node plane element based on variational principle containing Airy stress function. Engineering Computations, 27(4), 442-463 (2010)

[6] Wang Zhenke, WeiLelei, The equivalent spring calculation model of beam-columns with Retangle sections.

[7] Cen, S., Fu, X. R., Zhou, G. H., Zhou, M. J., and Li, C. F. Shape-free finite element method: the plane hybrid stress-function (HS-F) element method for anisotropic materials. Science China Physics, Mechanics \& Astronomy, 54(4), 653-665 (2011) 